\title{
The GEO 600 Gravitational Wave Detector Status, Research, Development
}

\author{
Albrecht Rüdiger and Karsten Danzmann for the GEO team \\ Max-Planck-Institut für Quantenoptik, Garching and Hannover, Germany
}

\begin{abstract}
The last few years have brought a great break-through in the quest for earth-bound detection of gravitational waves: at five sites, laser-interferometric detectors, of armlengths from 0.3 to $4 \mathrm{~km}$, are being built. These projects have in common that one prominent noise source, the shot noise, is reduced by the use of power recycling. By using advanced optical technologies early on, the German-British project GEO 600, although only intermediate in size $(600 \mathrm{~m})$, has good chances for a competitive sensitivity, at least with the first versions of the larger detectors. Particularly the use of the so-called signal recycling technique will allow to search for faint sources of only slowly varying frequency (pulsars, close binaries). The talk will describe the particular topology of the GEO 600 interferometer, characterized by the use of a four-pass delay line and signal recycling. The major noise sources, and the experimental effort aiming at their reduction, will be discussed. The current status of the construction of GEO 600 will be outlined (civil engineering, vacuum, optics). The research and development activities at the experimental sites (Garching, Glasgow, Hannover) will be given broad emphasis. First science runs of GEO 600, well in time with those of other ground-based interferometers, are expected in the year 2001.
\end{abstract}

\section{Introduction}

The construction of laser interferometers for the detection of gravitational waves is being pursued by various groups all over the world and, due to their particular preferences, their past history, and their funding, differing approaches have been developed. This diversity can, at the present stage, be seen as a great asset, as it will allow the community to develop and investigate a wide variety of promising schemes and evaluate their respective merits.

It is particularly in this field of research and development that a fruitful collaboration within the whole gravitational-wave community can and should be intensified.

This paper will give an account of the current status of the German-British project GEO 600 and elaborate on the various fields of research and development at the main centers of GEO activity: Hannover, Glasgow, and Garching. In many of these fields, the GEO groups have taken a leading role.

\section{The GEO 600 Concept}

At five sites, large-scale gravitational wave detectors using laser interferometry are being built and are nearing completion: at two sites in the US, interferometers of $4 \mathrm{~km}$ are being built (LIGO), the French-Italian collaboration VIRGO is 
building a $3 \mathrm{~km}$ detector near Pisa, and on a somewhat smaller scale, with $600 \mathrm{~m}$ and $300 \mathrm{~m}$ armlength: the German-British antenna GEO 600 near Hannover, and the Japanese antenna TAMA near Tokyo.

In the case of GEO 600, the smaller armlength was not a matter of choice, but one of necessity. The site (on grounds belonging to the University of Hannover) cannot accommodate a larger antenna, and the funds did not allow buying or leasing ground elsewhere. This paper will try to outline how this shortcoming in length is to be compensated, at least partially, by the application of advanced interferometric techniques.

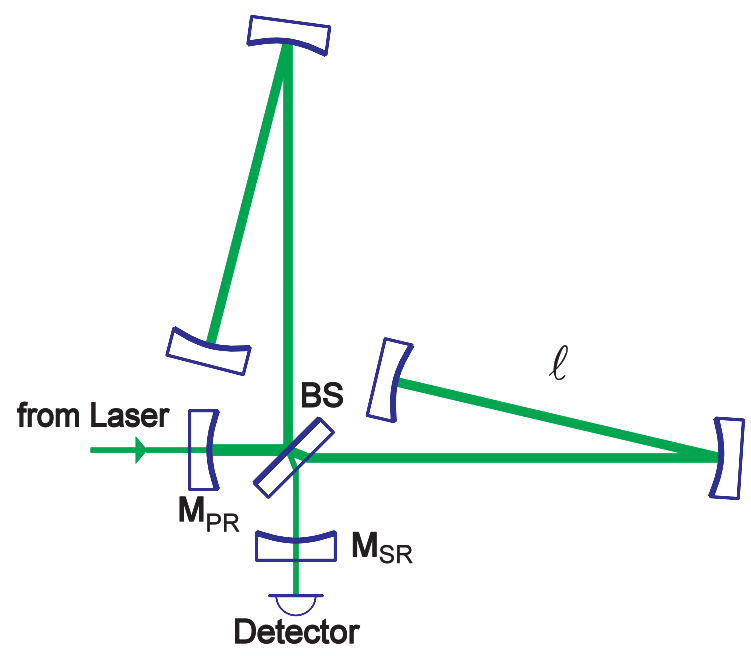

Fig. 1. DL 4 configuration of the GEO 600 interferometer. Two extra mirrors $\mathrm{M}_{\mathrm{PR}}$ and $\mathrm{M}_{\mathrm{SR}}$ for power and signal recycling.

support in a delay line made only of 'small' mirrors (the GEO 600 mirrors will nevertheless have a diameter of $18 \mathrm{~cm}$ ).

An outstanding characteristic of the GEO 600 concept is the use of 'dual recycling' $[1,2]$ : In addition to the scheme of power recycling, which is now standard in all the large detectors, a further mirror $\left(\mathrm{M}_{\mathrm{SR}}\right)$ is introduced in the output port, to allow a resonant enhancement of the sidebands that the gravitational wave produces from the carrier beam.

\section{Noise Contributions}

To obtain the projected sensitivity, extensive theoretical and experimental work is required to reduce the contributions of noise that would limit the sensitivity of GEO 600. These efforts (some are very similar to work going on at other 
institutions) are being carried out at Hannover, Glasgow, and Garching, with assistance also from the institutions at Cardiff and Potsdam.

Some of the more prominent noise sources (but by no means an exhaustive list) will be discussed below. Although these noise contributions are very serious, current technologies can reduce them to a level at which an interesting sensitivity of GEO 600 can be attained.

\subsection{Seismic Noise}

The influence from external mechanical vibrations, i.e. from seismic noise, is strongly reduced by a multiple pendulum suspension, indicated in Fig.2. With triple pendulum suspensions for the most critical optical components (mirrors, beam splitter), and double pendulums for the less critical components, GEO 600 is making a very ambitious effort, topped only by the even more extreme design of the VIRGO project with its seven pendulum stages. Installation at GEO has begun.

GEO's multiple pendulums are hung from a set of cantilever springs for better vertical isolation. These springs are supported by a top frame that itself is isolated via an encapsulated stack of 'rubber' and metal layers.

Underneath this stack, an active seismic control (feedback plus perhaps feedforward) will provide further isolation down to very low frequencies.

The forces to the test mass pendulum masses are applied from reaction masses that are similarly suspended, to avoid direct coupling from ground noise. The forces to the intermediate masses are applied via coil-and-magnet actuators. For the application of only very minute forces to the actual test masses (the lowest stage), an electrostatic scheme is being considered.

Work in this field was led by the Glasgow group, with contributions also from JILA (active isolation) and in close collaboration with the other GEO laboratories. The GEO pendulum design is a candidate for the suspension of the masses in the Advanced LIGO.

\subsection{Thermal Noise}

The thermal ('Brownian') noise of the suspended optical components can be split into two separate regimes: the internal vibrations of the solid suspended bodies (mirrors, beamsplitters), and the pendulation modes due to the pendulum suspension. Both must be kept as low as possible, to the very limit of current technology (or beyond).

The internal mechanical vibrations of the mirrors (the 'test masses') can be kept low by choosing materials of very high mechanical $Q$, such as pure fused silica, sapphire, or even silicon. (Note that the mirrors used in the DL4 scheme do not need to be transparent.) Elaborate methods of attaching the suspending 'wires' are required so as not to compromise the intrinsic $Q$. Glasgow and Hannover have worked out viable solutions, and $Q$ values of $5 \times 10^{6}$ and better have been achieved with fused silica mirrors. This internal noise is responsible for the rather shallow portion of the curve 'thermal noise' in Fig.3, above $50 \mathrm{~Hz}$. 
Also for the pendulum motion, extremely low-loss wires or fibers, and very elaborate techniques for attaching these 'wires' to the solid masses were developed, and very promising results were obtained, again by research carried out both at Glasgow and Hannover [3].

Low-loss silica fibers (or thin ribbons) were welded on to small silica prisms, and these were, in turn, attached to the mirrors using a bonding technique developed at Stanford, by 'hydroxy-catalysis bonding of silicates' [4]. This method turned out to be very successful. The measurements on these pendulums were done in close collaboration with the VIRGO group at Perugia. The pendulum thermal noise is the cause of the steep rise of the thermal noise curve as one goes to lower frequencies (in GEO 600 below, say, $30 \mathrm{~Hz}$ ).

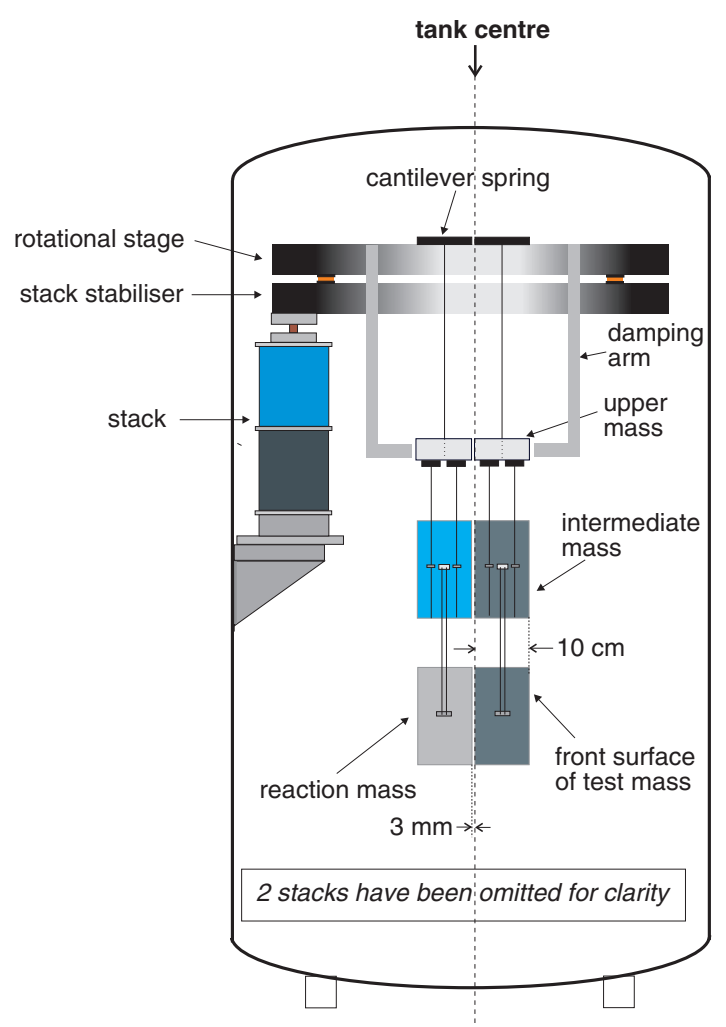

Fig. 2. Schematic of the GEO suspension. The actual test mass is on the right-hand side, forces are applied from equally quiet reaction masses, to avoid introducing ground noise.

\subsection{Laser Noise}

The development of the laser system for GEO 600 is mainly in the hands of Laser Zentrum Hannover (LZH). The master/slave system, with its highly stabilized Nd:YAG MISER as 'master' and a powerful slave, are now completed, $14 \mathrm{~W}$ are realiably available [5]. The master is already out at the site. Improved stabilization of the laser with respect to frequency and power is the goal of work going on at Hannover.

The geometrical noise of the beam, i.e. fluctuations in position, orientation, and shape of the beam, are greatly reduced by the use of 'mode-cleaners' first introduced by the GEO groups [6,7]. GEO 600 will use two in series, and intensive work was done at Glasgow. These mode-cleaners are the first units being optically tested, in the late months of 1999. Mirrors and seismic isolation are installed, and locking of the first stage has been achieved. 


\section{Shot Noise}

A very fundamental noise source is the shot noise produced by the 'graininess' of the detected light. Its effect decreases with the square root of the available light power. To reach sensitivities that make the detection of events from as far out as the Virgo cluster possible, light powers in the order of $10 \mathrm{~kW}$ are needed.

As these light powers cannot be supplied by today's lasers, the scheme of 'power recycling' will be applied in GEO 600, as in all other detector projects. The cavity composed of the Michelson interferometer and the power recycling mirror $\mathrm{M}_{\mathrm{PR}}$ is designed to resonantly enhance the light power by three orders of magnitude. Recycling gains in the order of 300 have been obtained [8] in the Garching 30-meter prototype.

\section{The GEO 600 Sensitivity}

Fig.3 shows the predicted sensitivity (i.e. the spectral density of the apparent strain noise) of GEO 600, resulting from the expected contributions of the various noise sources.

Having the signal sidebands resonate in the cavity formed by the Michelson interferometer as the one (albeit very complex) mirror, and the signal-recycling mirror $\mathrm{M}_{\mathrm{SR}}$ as the other, will make the signal response a rather complicated function of the GW frequency, which can be adapted to a variety of requirements.

On the left-hand side, the broadband operation of GEO 600 is shown, exhibiting a broad noise minimum around a frequency of $200 \mathrm{~Hz}$. The limiting effects are thermal noise between 40 and $200 \mathrm{~Hz}$ and shot noise from $300 \mathrm{~Hz}$ on upward.

By appropriate choice of the transmission of the 'signal recycling mirror', a narrowband operation is possible, and the antenna can (by microscopic positioning of the mirror $\mathrm{M}_{\mathrm{SR}}$ ) be tuned to a given frequency, e.g. to a known or expected GW source. This is indicated in the righthand diagram, showing a dip in the shot-noise contribution at $600 \mathrm{~Hz}$.

In this way, the limitation due to shot-noise can be reduced, if one is willing to "sacrifice" measuring bandwidth. There can be very good reasons to do that: for a nearly continuous-wave signal, as in the early stages of a binary inspiral, the signal frequency changes only very slowly, and narrow-banding at the 'response' level reduces noise much more effectively than narrow-banding at the dataanalysis level. But, of course, the thermal noise (intrinsic noise of the mirrors) does then limit the sensitivity.

\section{Interferometry}

A major subject of research at GEO 600 was the design, the analysis, and a critical assessment of various advanced interferometric techniques: dual recycling $[1,2]$ and resonant sideband extraction [9] appear to have great potential 

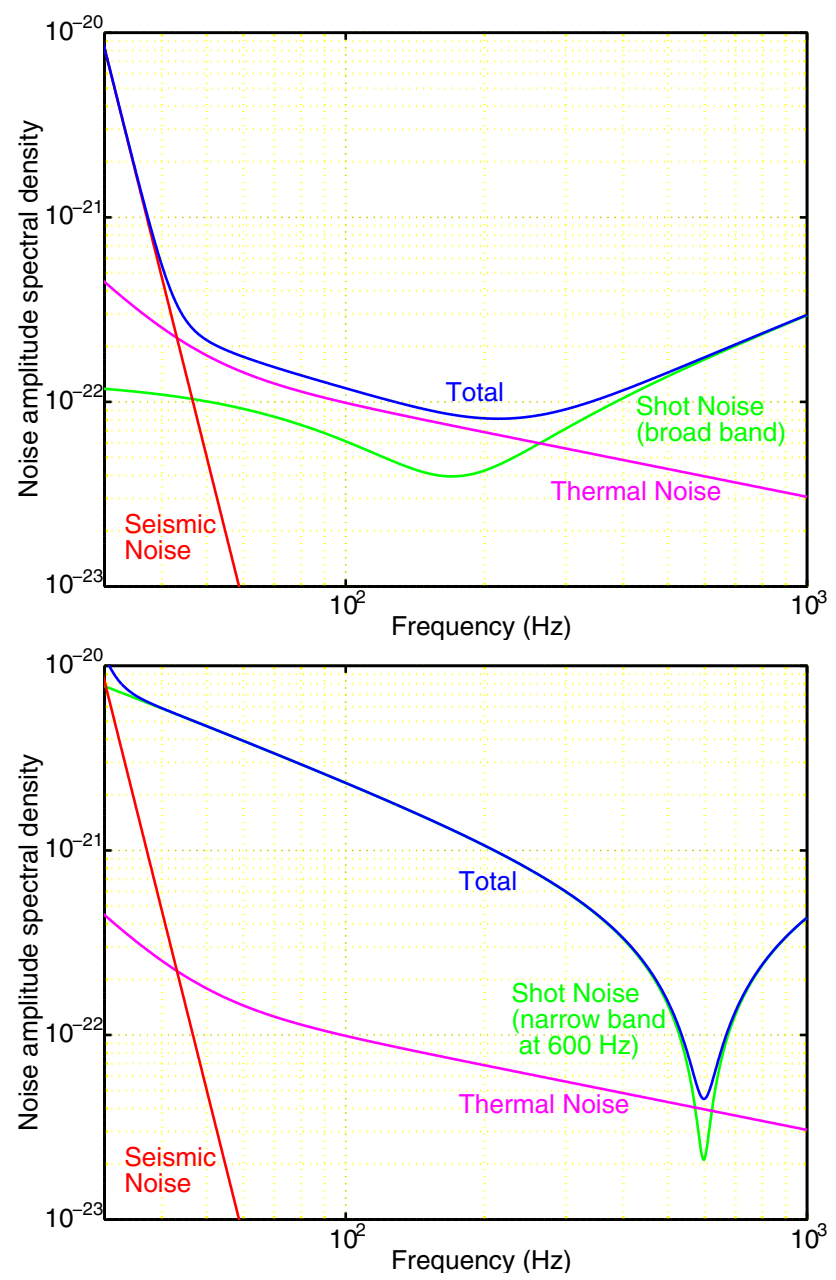

Fig. 3. Noise spectral density of GEO 600, shown for two operating modes: for broadband (top) and narrowband operation (bottom).

also for future large detectors. But also the feasibility of other interferometric schemes (e.g. Sagnac [10]) was explored.

Of the schemes investigated, it will be particularly the scheme of 'dual recycling' that will find application in GEO 600. Elaborate simulation programs were written (e.g. [11]), compared at a Workshop in Garching, and shared with colleagues from other groups.

At that workshop, also a variety of other software tools were presented to assist in the optical design [12] and development of controls [13] for advanced interferometry. These have been (or will be) made available to everybody in the community. 


\section{Civil Engineering}

GEO 600 was, from the beginning, a project that had to take into account a very low budget (the lowest of the world-wide community). Many of the features in GEO's implementation are thus dictated by budget considerations. This was, at times, a great burden, but it also gave an incentive for designing and developing very cost-efficient techniques that can become the basis also for future largescale detectors.

\subsection{Construction}

The construction of the central house and the end houses had been completed in 1997. The size of these buildings is at the lower limit, the accommodation of electronics and other equipment is scant, but sufficient. Some mechanical equipment is available at the site, but the main workshops are at the university site in Hannover.

The clean-room environment is a low-cost design which, however, turned out to be working very satisfactorily; a clean-room class 100 was readily achieved, and further improvement during critical work can be achieved by using laminarflow tents above the vacuum tanks.

The connecting trenches that house the vacuum tubes were finished even earlier. The trenches are open constructions, the sides consisting of steel panels driven into the ground. For weather protection, they are loosely covered with a corrugated steel roofing.

\subsection{Electronics and Data Management}

Most of the electronics is designed and built by the labs in Glasgow, Hannover, and Garching. Communication between the buildings will be exclusively via glass fiber, to avoid crosstalk. The data can be sent via direct radio communication to the University of Hannover for on-line monitoring and possible remote interaction, and from there via fast data links to Potsdam and Cardiff for analysis.

\section{The Vacuum System}

\subsection{Vacuum Pumps}

The good vacuum required in the whole apparatus is made possible by powerful turbo-molecular pumps that are situated in the central house and in the end houses. They will run continuously, i.e. also during measurements; the magnetic bearings allow a very quiet operation. 


\subsection{Vacuum Tubes}

GEO 600 has tried a very costeffective way of producing the vacuum tubing. The tube is made of pieces of $60 \mathrm{~cm}$ diameter stainless steel tubes of less than $1 \mathrm{~mm}$ thickness. The necessary stiffness against air pressure stems from a bellows-type convolution with a few $\mathrm{cm}$ period (see Fig.4). The tube is suspended from crossbars that traverse the trench.

\subsection{The Vacuum Achieved}

The tubes, wrapped in a $20 \mathrm{~cm}$ layer of rock wool, were first airbaked at $200{ }^{\circ} \mathrm{C}$ for one week, and then baked under vacuum for several more days. The vacuum achieved, in the range of $10^{-9} \mathrm{mbar}$, is by far sufficient. The tubes are supposed never to be let up to air again: they are shut off with gate valves when opening the vacuum tanks becomes necessary, as in the present phase of installation of suspensions.

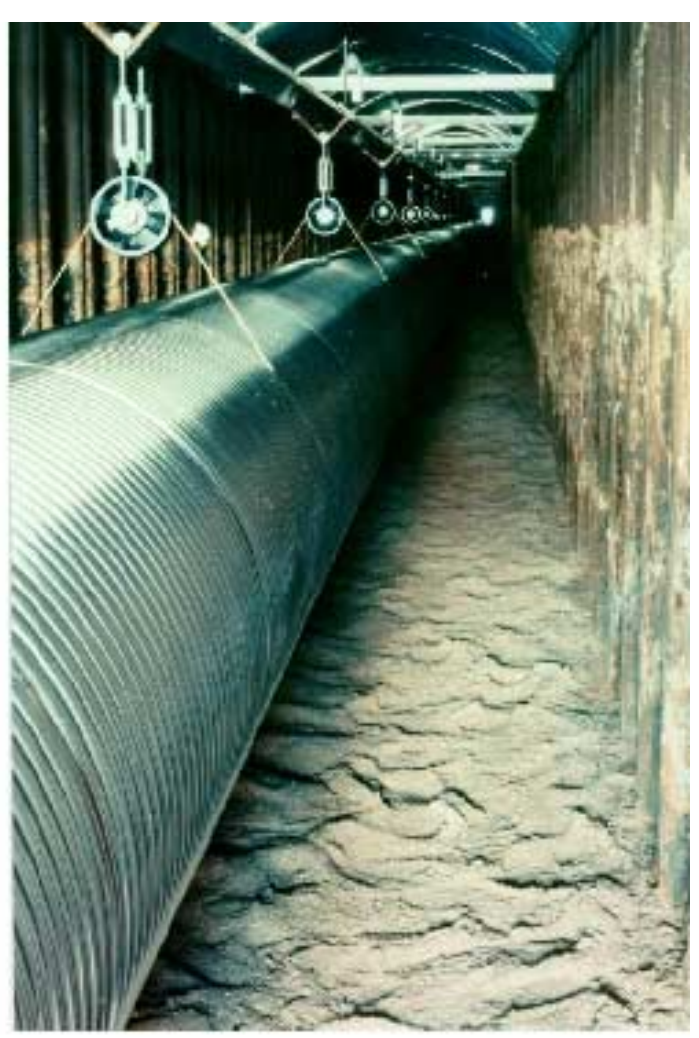

Fig. 4. The convoluted tubes during installation; with each new $4.5 \mathrm{~m}$ piece welded on, the tube was slid $4.5 \mathrm{~m}$ further into the $600 \mathrm{~m}$ long trench, using trolleys along the aluminum rail at the top.

\subsection{Vacuum Tanks}

The vacuum tanks (9 in the central house, one each in the end houses) have been leak-tested, baked out, and installed. The metal-and-rubber stacks, three per tank, are encapsulated in steel bellows to protect the vacuum. They are finished, most already installed. They will carry the top suspension structures, from which double or triple pendulums will carry the main optical components.

\section{The 30 Meter Prototype}

Many of the advanced interferometric concepts require verification and optimization by laboratory experiments. Such experimental development went on at the Glasgow $10 \mathrm{~m}$ prototype, as well as at the Garching $30 \mathrm{~m}$ prototype. It is the 


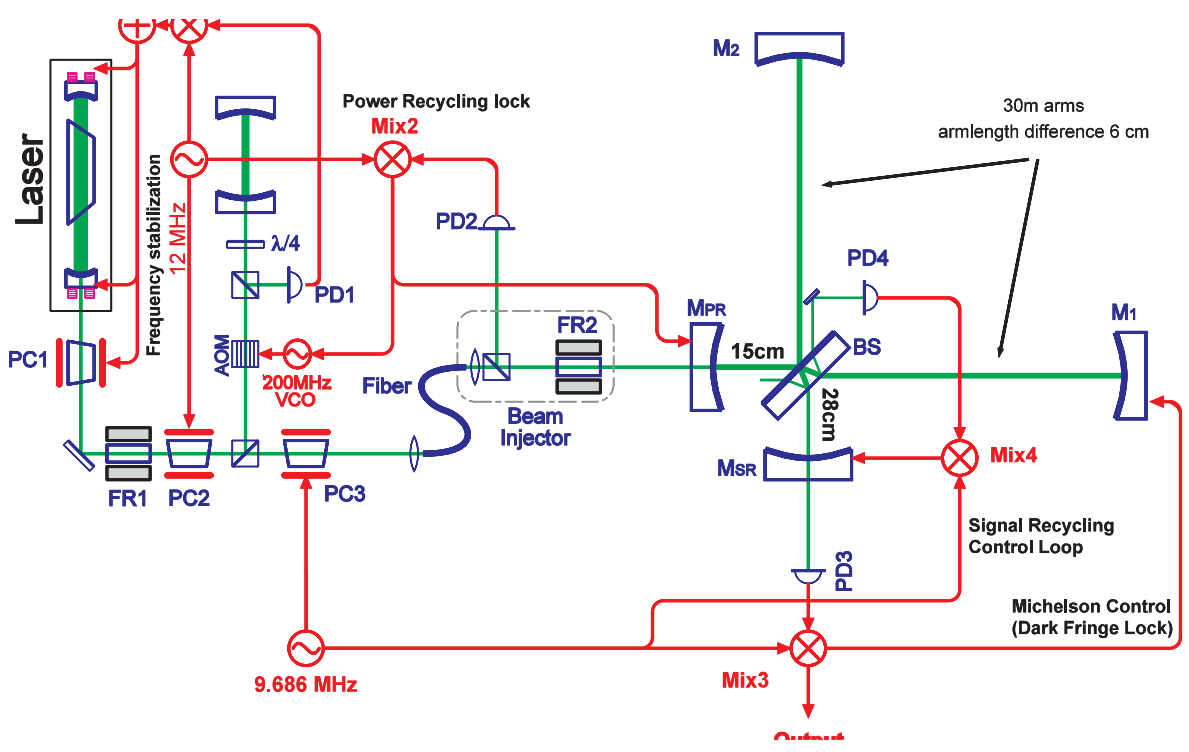

Fig. 5. The Garching $30 \mathrm{~m}$ prototype.

latter that will be treated here in some more detail. For a full discussion, see $[16]$.

The Garching $30 \mathrm{~m}$ prototype (Fig.5) was used for verifying the concepts of power and signal recycling, and for experimentally investigating the demands on the overall control system.

The laser (here still an Argon ion laser) is first stabilised against a reference cavity, and then against the power recycling cavity consisting of the interferometer and the mirror $\mathrm{M}_{\mathrm{PR}}$. A fully automatic alignment system for controlling 10 mechanical degrees of freedom provides the stability of the settings of the optical components [14]. The frontal ('Schnupp') modulation simplifies the read-out with which the Michelson interferometer is kept in lock, as compared with the previous concept of 'external modulation'.

Only after this set-up was sufficiently robust, the next step, the introduction of the signal recycling mirror $\mathrm{M}_{\mathrm{SR}}$, was started. Error signals for controlling its position are obtained with the same Schnupp modulation, using an additional photodetector, PD4. One big problem in such complicated interferometers is the fact that many of the control loops can work properly only after all other loops are locked. The operation of prototypes is essential in finding feasible schemes for this lock acquisition problem. The appropriate arm lengths between beam splitter and the four mirrors were chosen with the help of an elaborate simulation code, developed in close collaboration between Glasgow and Garching.

With all of this cautious preparatory work done, acquiring lock of the signal recycling loop was then surprisingly easy, and gave the expected enhancement of the signal response [15]. It furthermore gave evidence of the important effect of 'mode healing' when signal recycling is introduced: Only those transversal 
modes that are supported by the signal recycling cavity will be dominating at the output photo diode, which results in a contrast enhancement.

At the beginning [15], the output was still dominated by an excessive frequency noise contribution from the laser. Solving this problem gave a considerable improvement in sensitivity, but studies for further noise reduction are going on.

\section{Data Management}

An integral part of GEO 600 are the groups at Cardiff and Potsdam. In working on the theory of gravitational wave sources, they help to choose the right settings for the detector parameters. The choice of the data acquisition hardware and the development of the software is made in close collaboration with these groups. Strategies for sensitive and efficient signal detection are being developed both at Potsdam and Cardiff.

\section{Outlook}

The aim of GEO 600 is to have first science runs of the interferometer in the year 2001. This is well within the time when also other large detectors will begin taking data. It will be an exciting time to see gravitational wave astronomy to come about.

An interesting mix of theoretical, experimental, and technical problems is yet ahead of us, and these tasks could very well, and with benefit for all, be tackled in an international collaboration, by an exchange of ideas as well as of personnel. The GEO groups explicitly endorse any such collaboration.

\section{References}

1. B.J. Meers: Phys. Rev. D 38, 2317 (1988).

2. K.A. Strain, B.J. Meers: Phys. Rev. Lett. 66, 1391 (1991).

3. S. Rowan et al.: Phys. Lett. A 233, 303 (1997).

4. S. Rowan et al.: Phys. Lett. A 246, 471 (1998).

5. O.S. Brozek et al.: Proc. 18th Moriond Workshop, in press (1999).

6. A. Rüdiger et al.: Optica Acta 28, 641 (1981).

7. K. Skeldon et al.: Rev. Sci. Instrum. 67, 2443 (1996).

8. D. Schnier et al.: Phys. Lett. A 225, 210 (1997).

9. J. Mizuno et al.: Phys. Lett. A 175, 273 (1993).

10. J. Mizuno et al.: Opt. Comm. 138, 383 (1997).

11. J. Mizuno et al.: paper in preparation (1999).

12. R. Schilling: Optocad, paper in preparation (1999).

13. G. Heinzel: LISO, available from ftp.rzg.mpg.de/pub/grav/ghh/liso (1999).

14. G. Heinzel et al.: Automatic beam alignment in the Garching 30-m prototype of a laser-interferometric gravitational wave detector, Opt. Comm. 160, 321 (1999).

15. G. Heinzel et al.: Experimental Demonstration of a Suspended Dual Recycling Interferometer, Phys. Rev. Lett. 81, 5493 (1998).

16. G. Heinzel: Advanced optical techniques for laser-interferometric gravitational-wave detectors, Thesis, Univ. Hannover (1999); available as MPQ 243. 